\title{
Entomological Assessment of the Impact of Long- term Mass Administration of Ivermectin in Onchocerciasis - Endemic Communities in Kaduna State, Nigeria.
}

\section{Sunday Isiyaku ( $\sim$ sisiyaku@sightsavers.org )}

Sightsavers https://orcid.org/0000-0003-3839-5813

William Adamani

Sightsavers

Hamill Louise

Sightsavers

Iliya Shehu Ndams

Ahmadu Bello University

Kogi Ezekiel

Ahmadu Bello University

Alphonsus Kal

The Carter Center

James Gazama

Nigerian Institute for Trypanosomiasis Research

Ishaya Haruna Nock

Ahmadu Bello University

\section{Research}

Keywords: Assessment, Blackfly, Entomological, elimination, Ivermectin, Onchocerciasis, Simulium

Posted Date: September 2nd, 2021

DOI: https://doi.org/10.21203/rs.3.rs-840823/v1

License: (c) (i) This work is licensed under a Creative Commons Attribution 4.0 International License. Read Full License 


\section{Abstract}

Background: Onchocerciasis constitutes a major public health burden in endemic communities and areas because of its dermal and ocular manifestations. Nigeria accounts for the highest burden of the disease globally. Long term mass distribution of ivermectin has been recommended by the World Health Organization as a potent strategy in halting the transmission of the disease in endemic communities.

Aim: The aim of this study was to evaluate the prevalence of infective blackflies after 17 years of community-based treatment with ivermectin in two onchocerciasis foci in Kaduna State, Nigeria.

Method: Blood seeking female flies were collected using human landing capture from two breeding sites (River Galma and Gurara) in Lere and Kagarko LGAs of Kaduna State in 2010 and 2011. The fly heads and bodies, in pools of 100, were analysed using Polymerase Chain Reaction (PCR)-ELISA specific for $O$. volvulus DNA.

Results: A total of 24,937 blackflies were caught during two-year catching period. The prevalence of infective flies reduced from $0.15 \%$ to $0 \%$ at River Galma and $0.08 \%$ to $0.04 \%$ at river Gurara in 2010 and 2011 catching period. Some pools containing the bodies of the flies were also positive for Onchocerca volvulus.

Conclusion: The presence of $O$. volvulus in the heads and bodies of the black flies collected confirmed that there was ongoing transmission of onchocerciasis in Kaduna State, Nigeria during the period of survey. There is continued need for mass distribution of ivermectin in Kaduna in order to halt the transmission.

\section{Background}

Onchocerciasis is a vector-borne parasitic disease caused by the filarial nematode Onchocerca volvulus and transmitted by Simulium species. According to WHO, an estimated 217.5 million people live in areas at risk of onchocerciasis, the vast majority in sub-Saharan Africa. This estimate does not include low transmission areas that still need to be mapped [1]. The main complications of this infection are severe eye disease that lead to blindness and skin disease characterized by papular or hypo-pigmented lesions and intense itching. It is the microfilariae (mf) which drive this pathology.

The main strategy for combatting onchocerciasis is mass ivermectin (Mectizan ${ }^{\circledR}$ ) distribution. Following the discovery of ivermectin and its subsequent donation by Merck and Co, African control programmes adopted mass ivermectin treatment as their main control strategy, using community-directed treatment (CDTi) to distribute [2]. Ivermectin (Mectizan ${ }^{\circledR}$ ) is a potent microfilaricide that has limited effect on the viability and reproductive capabilities of adult onchocercal worms. Thus, repeated treatment is needed in order to suppress the manifestations of the infection over time and limit transmission. In 2009 the first evidence of elimination of the parasite from African foci using ivermectin alone emerged from Mali and Senegal [3]. This led to a wider evidence gathering effort by the African Programme for Onchocerciasis 
Control (APOC), and as evidence supporting the feasibility of onchocerciasis elimination in Africa using ivermectin alone mounted, countries switched the focus of their programmes from control to elimination. In 2020, evidence of onchocerciasis elimination through annual mass drug administration was documented in Plateau and Nasarawa states in Nigeria [4].

In Nigeria, Onchocerciasis is endemic in 31 of the 36 States of the country including the Federal Capital Territory of Abuja. Repeated annual treatment with ivermectin commenced in 1990 and scaled up by 1997 in most of the endemic communities after undertaking rapid assessment of communities to be treated using Rapid Epidemiological Mapping of Onchocerciasis (REMO) [5].

The treatment of onchocerciasis commenced in Kaduna State with the clinical trial of ivermectin in Lere LGA in 1989. This was carried out in collaboration with Guinness Ophthalmic Unit, University of London, World Health Organisation and Sightsavers. Treatment was later expanded to Birnin Gwari and Kauru LGAs of Kaduna State in 1990 [6]. The success of the clinical trial, which ended in 1991, set the stage for expansion of treatment to other LGAs in the state. Over the years, treatment for onchocerciasis increased from 6,149 people treated in 1989 to over 1.9 million people in 2012.

Wide-spread treatment with ivermectin has been ongoing at large scale in endemic LGAs in Kaduna State since 1997. Therefore, monitoring of both human and entomological indices of transmission is essential to determine the impact of ongoing treatment, and collect adequate entomological data to inform the design and implementation of future stop-MDA surveys. The World Health Organisation (WHO) guidelines on stopping onchocerciasis MDA stipulate that fly-catching and serological surveys must be conducted at the transmission zones before stop- MDA is implemented [7]. Previous epidemiological studies by Tekle et al in 2008 but published in 2012 reported zero cases of onchocerciasis in the endemic communities in Kaduna State [6]. In the present study, we present the results of entomological assessment of effects of 17 years mass distribution of ivermectin in two onchocerciasis foci in Kaduna State, Nigeria.

\section{Materials And Methods}

\section{Study Area}

The study was carried out in Kaduna State $\left(10^{\circ} 33^{\prime} \mathrm{N} ; 7^{\circ} 75^{\prime} \mathrm{E}\right)$ located in the middle belt of Nigeria with a projected population of 7.31 million (National Population Commission, 2006). The state shares boundaries with Katsina and Kano States in the north, Plateau State to the east, Nassarawa State to the southeast, Niger State to the west and Federal Capital Territory, Abuja (FCT) to the south. Kaduna State is intersected by two main rivers, Kaduna and Gurara rivers. Other rivers bordering on the state include Kogum, Matsirga and Galma, in addition to several streams and rivulets. All of these provide opportunities for irrigation and fishing. The fast flowing rivers and their tributaries also serve as breeding sites for black fly. 
The study was carried out in two onchocerciasis endemic foci, Kudaru (Lere LGA) and Gantang (Kagarko LGA) both located in Kaduna State "Additional file 1: Figure S1".

\section{Ethical Clearance}

Ethical approval was sought and obtained from the Kaduna State Ministry of Health before the commencement of the study (Ref No. MOH/ADM/122/VOLII/560). Verbal informed consent was obtained from the local government authorities, community leaders and fly collectors. The fly collectors were treated with ivermectin before and after completion of the study.

\section{Collection of Blackflies}

The entomological evaluation was carried out over two wet seasons in 2010 and 2011 coinciding with the period of high density of flies and onchocerciasis transmission in Nigeria. Blood seeking female blackflies were collected using the human landing method $[7,8]$ from two main breeding sites in each location. Four fly collectors worked simultaneously between $07.00 \mathrm{hr}$ and 18:00hr for three consecutive days weekly between June and October in 2010 and 2011. The samples were preserved in 30ml bottles containing $100 \%$ isopropanol and stored at $4^{\circ} \mathrm{C}$ until use.

\section{PCR-ELISA Pool Screening Assay}

The heads and bodies were pooled (100 heads/bodies per pool) and subjected to DNA extraction and PCR-ELISA Pool Screening Assay using protocol earlier described by Unnasch [9]. The DNA extraction and PCR amplification targeting 0-150 repeats were carried out as previously described by [9, 10]. The ELISAbased detection of the amplified PCR products was carried out with a Spectra Max Microplate Reader Model Vmax (EMax Endpoint ELISA Microplate Reader). A pool on a particular plate is positive when the optical density of the well is greater than the mean plus 3 standard deviation of the 10 negative controls in the plate as read from micro plate reader [9].

\section{Statistical Analysis}

The results obtained from PCR - ELISA Pool screening Assay were subjected to statistical analysis to calculate the infective, infection and confidential intervals using PoolScreen software version 2.0 [11, 12, 13].

\section{Result}

\section{The Biting rates of Simulium collected in Kudaru and Gurara foci}

A total of 24,973 Simulium damnosum adult flies were collected in two wet seasons of year 2010 and 2011. More flies were collected in 2011 (4, 691 in Kudaru; 10,600 in Gurara) as compared with 2010 (2,093 in Kudaru; 7,553 in Gurara). There is a no significant difference in the number of flies collected in both sites across the years $(P>0.05)$. However, the fly population varied significantly $(P>.05)$ with more flies collected around River Gurara $(18,153)$ as compared with River Galma $(6,784)$. The average hourly catch per site ranged from 0.87 to 4.42 "Additional file 2: Table S1". 
The results of the monthly biting Monthly Biting Rate (MBR) are presented in "Additional file 2: Table S2". The results showed the highest biting rate was recorded in July in both sites in 2010 and 2011 (1,089 and 1,713 flies per man per month Gurara and 305 and 1141 flies per man per month in Kudaru in 2010 and 2011 respectively) "Additional file 2: Table S2". There was significant difference in biting rates recorded across the catching months in both sites in 2010 and $2011(P<0.05)$.

\section{The infective and infection rates of Simulium damnosum s.l collected in Kudaru and Gurara foci}

A total of 13 and 19 pools containing the heads and the bodies of the flies were positive for 0 . volvulus from pools of 250 heads and pools of 250 bodies. In Galma, the infective rate was $0.15(0.03-0.45)$ in 2010 and $0(0-0.04)$ in 2011. In Gurara, the infective rate was $0.08(0.03-0.18)$ in 2010 and 0.04 (0.010.10 ) in 2011 (Table 3). The infective rate in 2010 and 2011 was statistically significant in River Galma $(P<0.05)$ while there was no significant difference in infectivity rate in both catching years in River Gurara $(P>0.05)$.

The prevalence of infection (as estimated by number of body pools positive for $O$. volvulus) in River Galma is $0.1 \%$ in 2010 and $0.19 \%$ while in River Gurara, the prevalence of infection is $0.04 \%$ and $0.06 \%$ in 2010 and 2011 respectively. The was no significant difference in the infection in the two foci in both catching years $(P>0.05)$ "Additional file 2: Table S3".

\section{Discussion}

The earlier studies in Kaduna in 1987 described the state as one of the most endemic foci of onchocerciasis in Nigeria [6]. This necessitated the selection of Kaduna as one of the sites for clinical trials of ivermectin in Nigeria. By 2010, the endemic communities in Kaduna had received 17 annual rounds of MDA. Contrary to the report of epidemiological assessment conducted in 2008 which reported zero prevalence of $O$. volvulus in human population in the communities surveyed at the study area (6), the results of PCR-ELISA showed positive pools for both heads and bodies which indicated an ongoing transmission of the disease and uptake of the parasite from human population.

Entomological assessment has been adjudged as the most critical tool in estimating the real-time assessment of transmission in the endemic communities $[14,15,16]$. The positive pools of the bodies of the flies confirm that the flies have access to infected individuals in the community possibly with moderate to high microfilarial load. The detection of $L 3$ larvae in the head pools using $O$. volvulus specific PCR-ELISA also negates the argument that the parasite found in the flies may be animal parasite $(O$. ochengi).

Entomological studies on cytotaxonomic identification of members of Simulium damnosum complex present in the study area had earlier confirmed localized breeding of the black flies in the area [17]. The high population density and biting rates recorded in the present results also corroborate the earlier report. It is most unlikely that the flies collected during the study were migratory. Therefore, the transmission in the area is plausibly localized not migratory/importation. While the factors accounting for the 
discrepancies in the results of epidemiological and entomological surveys remained unclear and could not be established in the present study, the observation in the discrepancies justifies the need for both entomological and epidemiological surveys to be conducted simultaneously, most importantly in the current context of elimination. This will provide insightful and comprehensive data to guide the researchers and policy makers on decision on stop MDA.

Even though, the results of the Pool screening PCR Assay indicated drastic reduction in infective rates in both foci (to zero in Galma) in 2011 as compared with 2010, the fact the positive pools of body segment of the flies indicate that active uptake of microfilaria by the flies from human population is ongoing in the study area. The results are consistent with results from other areas where MDA had been conducted for a similar period of time indicating reduction but not interruption in Cameroon $[18,19]$.

The significant variation in population and biting densities of black flies in River Galma and River Gurara are expected. The prolific breeding of black flies in Africa has been associated with presence of rocks, vegetation, speed of the water and other physico-chemical parameters of the river [20.14,21]. The presence of the factors are more feasible at River Gurara and thus accounting for high productivity of the breeding site all year round. The number of flies caught along River Galma each year were below the recommended 6,000 per transmission zone, recommended by WHO for stop MDA surveys [4]. This finding is important for planning future monitoring and evaluation or onchocerciasis MDA in Kaduna; additional catching hours, sites, or time would be required to meet the 6,000 fly threshold.

\section{Conclusion}

The results of the entomological assessment demonstrated ongoing transmission of onchocerciasis as evaluated in 2010 and 2011 in two endemic foci of Kaduna State, Nigeria despite 17 years of mass distribution of ivermectin. There is therefore need for continued annual distribution of ivermectin with good therapeutic coverage in order to halt the transmission in the study area.

\section{Declarations}

\section{Acknowledgement}

The communities and fly collectors and The Carter Center for the use of their laboratory in Nigeria for the analysis.

\section{Ethics approval and consent to participate}

Consent for publication

\section{Availability of data and materials}

\section{Competing interests}




\section{Funding}

\section{Authors' contributions}

\section{References}

1. WHO Weekly epidemiological record 08 November 2019, vol 94, No 45, 2019, 91, 513 - 534. _https://www.who.int/wer/2019/wer9445/en/_

2. Amazigo U. The African Programme for Onchocerciasis Control (APOC) Ann Trop Med Parasitol. 2008; 102(Suppl 1):19-22

3. Diawara L, Traore MO, Badji A, Bissan Y, Doumbia K, Goita SF, et al. Feasibility of onchocerciasis elimination with ivermectin treatment in endemic foci in Africa: first evidence from studies in mali and senegal. PLoS Negl Trop Dis. 2009, 3: e497-10.1371/journal.pntd.0000497.

4. Richards, F. O., Eigege, A., Umaru, J., Kahansim, B., Adelamo, S., Kadimbo, J., et al. (2020). The Interruption of Transmission of Human Onchocerciasis by an Annual Mass Drug Administration Program in Plateau and Nasarawa States, Nigeria. The American journal of tropical medicine and hygiene, 102(3), 582-592. https://doi.org/10.4269/ajtmh.19-0577

5. Oyibo W, Fagbenro-Beyioku AF. Effect of repeated community-based ivermectin treatment on the intensity of onchocerciasis in Nigeria. Rural and Remote Health 2003; 3: 211. Available: www.rrh.org.au/journal/article/211

6. Tekle A. H., Elhassan, E., Isiyaku, S., Amazigo, U. V., Bush, S., Noma, M., et al. (2012). Impact of longterm treatment of onchocerciasis with ivermectin in Kaduna State, Nigeria: first evidence of the potential for elimination in the operational area of the African Programme for Onchocerciasis Control. Parasites \& Vectors, 5:28.

7. World Health Organisation WHO (2016). Guidelines for stopping mass drug administration and verifying elimination of human onchocerciasis: Criteria and procedure. WHO/HTM/NTD/PCT/2016.1

8. Rodriguez-Perez, M. A., Lilley, B. G., Dominguez-Vazquez, A., Segura-Arenas, R., Lizarazo-Ortega, C., Mendoza-Herrera, et al. (2004). Polymerase Chain Reaction Monitoring of Onchocerca volvulus tin two endemic states in Mexico. American Journal of Tropical Medicine and Hygiene, 70(1): 38-45.

9. Walsh, J. F., Davies, J. B., Le Berre, R. and Garms, R. (1978). Standardization criteria for assessing the effect of Simulium control in Onchocerciasis control programmes. Transactions of the Royal Society for Tropical Medicine and Hygiene, 72: 675-676.

10. Unnasch, T. R. (2011). Complete Protocol for 0-150 PCR Analysis of Blackflies for O. volvulus infection. Protocol Adapted for African Black flies.

11. Katholi, C. R., Toe, L., Merriweather, A, and Unnasch, T. R. (1995). Determining the prevalence of Onchocerca volvulus infection in vector populations by Polymerase Chain Reaction screening of pools of black flies. Journal of Infectious Disease, 172:1414-1417

12. Unnasch, T. R. and Meredith, S. E. O. (1996). The use of degenerate primers in conjunction with strain of and species oligonucleotides to classify Onchocerca volvulus. Clapp J.P. ed. Species Diagnostic 
Protocols: PCR and Other Nucleic Acid Methods. Totowa NJ: Humana Press, 239 - 303.

13. Yameogo, L., Toe, L., Houghard, J. M., Boatin, B. A. and Unaasch, T. R. (1999). PoolScreening Polymerase Chain Reaction for estimating the prevalence of Onchocerca volvulus infection in Simulium damnosum s.l: results of a field trial in an area subject to successful vector control. American Journal of Tropical Medicine and Hygiene, 60:124-128.

14. Adeleke, M.A., Sam-Wobo, S.O., Olatunde, G.O., Akinwale, O.P., Ekpo, U.F, Mafiana, C.F (2011). Bioecology of Simulium damnosum Theobald complex along Osun River, Southwestern Nigeria. Journal of Rural and Tropical Public Health 10: 39-43

15. Rodríguez-Pérez M.A., Adeleke M.A, Rodríguez-Luna I.C, Cupp E.W, Unnasch,T.R (2014). Evaluation of a Community-Based Trapping Program to Collect Simulium ochraceum sensu lato for Verification of Onchocerciasis Elimination. PLoS Neglected Tropical Diseases 8(10), e3249. doi:10.1371

16. Oforka, L.C., Adeleke, M.A , Anikwe, J.C., Hardy N., Mathias, D., Makanjuola, W., Fadamiro, H (2020), Biting rates and Onchocerca Infectivity status of black flies from the Simulium damnosum complex (Diptera: Simuliidae) in Osun State, Nigeria, Journal of Medical Entomology 57(3): 901-907

17. Mafuyai, H.B., Post, R.J., Vajime, C.G. \& Molyneux, D.H. 1996. Cytotaxonomic identification of the Simulim damnosum complex (Diptera: Simuliidae) from Nigeria, Tropical Medicine and International Health 1: 775-7854

18. Katabarwa M.N., Eyamba A., Nwane P., Enyong P., Kamgno J., Kueté T., et al. (2013) Fifteen years of Annual Mass Treatment of Onchocerciasis with Ivermctin have not interrupted Transmission in the West Region of Cameroon. Journal of Parasitological Research, Vol 2013, Article ID 420928.

19. Katabarwa M. N., Eyamba A., Nwane P., Enyong P., Yaya S., Baldiagai J., Madi T. et al. (2011) Seventeen years of annual distribution of ivermectin has not interrupted onchocerciasis transmission in North Region, Cameroon. American Journal of Tropical Medicine and Hygiene 85, 1041 - 1049.

20. Ibeh O. O., Nwoke B. E. B., Adegoke J. A. (2007). Distribution and Ecology of breeding sites of Simulium damnosum s.I in Southeastern Primary health Zone of Nigeria. Nigerian Journal of Parasitology, 28(1): 32-8.

21. Oforka, L.C., Adeleke, M.A., Anikwe, J.C ., Makanjuola, W.A (2019): Population fluctuations and effect of climatic factors on the relative abundance of Simulium damnosum complex (Diptera: Simulidae), Environmental Entomology: 48 (2): 284-290

\section{Supplementary Files}

This is a list of supplementary files associated with this preprint. Click to download.

- AdditionalFile1.docx

- AdditionalFile2.docx

- GraphicAbstract.jpg 